\title{
Evaluation of Epigallocatechin-3-gallate Modified Collagen Membrane and Concerns on Schwann Cells
}

\author{
Chenyu Chu, ${ }^{1}$ Jia Deng, ${ }^{1}$ Cong Cao, ${ }^{2}$ Yi Man, ${ }^{1,3}$ and Yili Qu ${ }^{1}$ \\ ${ }^{1}$ State Key Laboratory of Oral Diseases, West China Hospital of Stomatology, Sichuan University, Chengdu 610041, China \\ ${ }^{2}$ China-Japan Friendship Hospital, Department of Stomatology, Beijing 100029, China \\ ${ }^{3}$ Department of Oral Implantology, West China Hospital of Stomatology, Sichuan University, Chengdu 610041, China
}

Correspondence should be addressed to Yi Man; manyi780203@126.com and Yili Qu; qqyili@126.com

Received 17 April 2017; Revised 5 June 2017; Accepted 5 July 2017; Published 15 August 2017

Academic Editor: Zetao Chen

Copyright (c) 2017 Chenyu Chu et al. This is an open access article distributed under the Creative Commons Attribution License, which permits unrestricted use, distribution, and reproduction in any medium, provided the original work is properly cited.

Collagen is an essential component of the extracellular matrix (ECM) and is a suitable material for nerve repair during tissue remodeling for fracture repair. Epigallocatechin-3-gallate (EGCG), an extract of green tea, shows various biological activities that are beneficial to nerve repair. Here, we developed modified collagen containing different concentrations of EGCG $(0.0064 \%, 0.064 \%$, and $0.64 \%$, resp.) to induce Schwann cell proliferation and differentiation. Cell Counting Kit- 8 test, live/dead assay, and SEM showed that collagen cross-linked by EGCG induced Schwann cell proliferation. Real-time polymerase chain reaction, enzymelinked immunosorbent assay, and Western blotting revealed that EGCG-modified collagen induced Schwann cell differentiation and downregulated reactive oxygen species (ROS) levels by downregulating the MAPK P38 signaling pathway. Our results indicate that collagen cross-linked with an appropriate concentration of EGCG induces the proliferation and differentiation of Schwann cells. The EGCG-modified collagen membrane may be applicable for nerve repair and guided tissue regeneration applications.

\section{Introduction}

Peripheral nerve regeneration is in a debilitating condition for which new bioengineering solutions are needed [1]. Autografting is the current gold standard treatment for nerve repair but is limited by the availability of expendable donor nerves, resulting in a second injury with the loss of sensation at the donor site $[2,3]$. Moreover, the nervous system is involved in bone remodeling after bone fracture [4]. It regulates bone regeneration by releasing related peptides, such as calcitonin gene-related peptide, neuropeptide $\mathrm{Y}$, and intestinal peptide [5]. Thus, nerve regeneration critically influences the success of guided tissue regeneration (GTR) treatment.

Recently, GTR biomaterials and cells have been developed for nerve repair. Extracellular matrix (ECM) molecules and Schwann cells (SCs) are important components of peripheral nerve repair. Armstrong et al. showed that ECM molecules affected SC behaviors, including attachment, proliferation, and secretion of neurite-promoting factors by SCs on a nerve conduit polymeric material in vitro.
Collagen is an essential component of the ECM, shows excellent biocompatibility, and is beneficial for a series of cellular behaviors including cell adhesion, proliferation, migration, and differentiation [6-8]. Collagen is widely used in nerve repair. Macaya et al. investigated the proproliferative effect of injectable collagen-based hydrogels by incorporating fibroblast growth factor-2 on astrocytes, which plays an important role in the healing of spinal cord injury [9]. Another study by Cui et al. involved the use of collagen scaffolds incorporated with the neurocytokines ciliary neurotrophic factor and basic fibroblast growth factor to bridge a nerve gap [10]. Moreover, Sadtler et al. applied an ECM-based collagen membrane to guide tissue regeneration by shaping the immune microenvironment. These studies indicated that an ECM-based collagen membrane mediates macrophages and $\mathrm{T}$ helper cells to promote systemic and local proregenerative immune responses [11]. Additionally, collagen was shown to promote nerve reconstruction. However, as a foreign material to the body, pure collagen may induce inflammatory responses, greatly limiting its biomedical applications. 
Epigallocatechin-3-gallate (EGCG) is an important component of tea and has been reported to have advantageous biological activities including antioxidant [12] and antiinflammatory effects $[13,14]$. Previous studies have suggested that EGCG protects nerve cells from oxidative-radical-stressinduced apoptosis [15], has anti-inflammatory and neuroprotective potential [16], modulates neurological function [17], activates nerve growth factors to induce neuritogenesis and reduce neuropathic pain after chronic constriction nerve injury [18], and stimulates regeneration of myelinated axons and minimizes histomorphological alterations caused by crush injury of the sciatic nerve [19].

Thus, EGCG-treated collagen may be useful in nerve repair and GTR [20, 21]. In this study, we fabricated an EGCG-modified collagen scaffold to accelerate tissue repair, trigger SC proliferation and differentiation, and induce secretion of nutrient growth factors. Cell viability, the levels of nutrient factors, including nerve growth factor (NGF) and brain-derived neurotrophic factor (BDNF), and the expression of Krox-20 in RSC96 cells were also measured to assess the biological properties of EGCG-treated collagen membranes.

\section{Methods and Materials}

2.1. Materials. Each type I collagen membrane (Zhenghai Biotechnology, Shandong, China) was commercially available and was prepared in a size of $10 \mathrm{~mm}$ diameter. The collagen membrane was made of natural and untreated ECM that maintains its structure and characteristics well. EGCG was obtained commercially (Jiang Xi Lv Kang Natural Products, Jiang Xi, China). All solvents and chemicals were of analytical grade and used without further purification.

2.2. Fabrication of EGCG-Modified Collagen. EGCG was dissolved in deionized water to prepare EGCG solutions of $0.64 \%, 0.064 \%$, and $0.0064 \%(\mathrm{w} / \mathrm{v})$ according to the previous study [22]. Then, collagen membranes were immersed in different concentrations of EGCG at room temperature for $1 \mathrm{~h}$ to fabricate the EGCG-modified collagen membranes. After being immersed, the resulting collagen membranes were rinsed three times with deionized water and then freeze-dried overnight. A control group was incubated with deionized water under the same experimental conditions.

2.3. Cell Viability. Cell Counting Kit-8 (Dojindo Laboratories, Kumamoto, Japan) was applied to detect cell viability. The cells were cultured in DMEM with $10 \%$ FBS. All the pure and the EGCG-modified collagen membranes were placed in 48 well plates where RSC96 cells of $10^{4}$ /well were seeded. After seeding, $10 \%$ of CCK-8 solution was added to each well, and then the 48 well plates were continuously incubated at $37^{\circ} \mathrm{C}$ for $2 \mathrm{~h}$. After incubation, the absorbance of the solution was read at $450 \mathrm{~nm}$.

2.4. Live/Dead Cell Assay. To further investigate the viability of cells, a live/dead cell assay kit (Dojindo Laboratories, Kumamoto, Japan) was applied according to the manufacturer's instructions. RSC96 cells were cultured on various collagen membranes for 5 days before being examined. Membranes with cells seeded were washed with 1x PBS for $5 \mathrm{~min}$ and incubated with Calcein-AM $2 \mu \mathrm{M}$ plus EthD-1 $4 \mu \mathrm{M}$ for $15 \mathrm{~min}$ at $37^{\circ} \mathrm{C}$ in the dark. After incubation, the resulting samples were then washed with 1x PBS for $5 \mathrm{~min}$ and then analyzed by an Inverted $\mathrm{Ti}-\mathrm{E}$ microscope (Nikon, Japan).

2.5. Scanning Electron Microscope (SEM) Observation. SEM (S-800, HITACHI, Tokyo, Japan) with an accelerating voltage of $25 \mathrm{kV}$ was used to observe the morphologies of collagen membranes. In order to achieve enough electrical conductivity, all the samples were coated with an ultrathin layer (300 $\AA$ ) of $\mathrm{Au} / \mathrm{Pt}$ in an ion sputter (E1010, HITACHI, Tokyo, Japan).

2.6. Immunostaining of Cells. For immunofluorescence staining of F-actin (cytoskeleton), Krox-20 (differentiation marker of Schwann cells), and nucleus, the cells on the samples were fixed in $2 \%$ paraformaldehyde in PBS for $5 \mathrm{~min}(\mathrm{pH}$ 7.4) followed by being washed three times in PBS ( $5 \mathrm{~min}$ ). Then, the samples were pretreated with $1 \%$ bovine serum albumin in PBS containing 0.1\% Triton X-100 (1 h) and then incubated in 1\% Tween 20 for $20 \mathrm{~min}$. After incubation, the samples were given a $5 \mathrm{~min}$ wash in PBS, and then cells were covered by sufficient stain solution and incubated for 1-5 min in the dark. All of antibodies were purchased from Santa Cruz Biotechnology (Texas, USA).

After removing the stain solution by washing the cells 2-3 times in PBS, fluorescent images were collected.

2.7. Neurotrophins Determination by Real-Time Polymerase Chain Reaction (RT-PCR) and Enzyme-Linked Immunosorbent Assay (ELISA). RSC96 cells were seeded on the pure collagen and collagen loaded with EGCG of different concentrations and then incubated for 7 and 14 days. Total RNA was extracted with TRIZOL reagent and reverse transcribed using the mRNA Selective PCR kit. Rat NGF and BDNF cDNA were amplified by real-time PCR using the SYBR Green PCR kit. The primer sequences used for the realtime PCR are shown in Table 1. Moreover, levels of growth factors in the supernatants were determined using an ELISA kit. According to the manufacturer's instructions, the levels of these neurotrophins were analyzed using an Opt ELISA kit (BD Biosciences, San Jose, CA, USA). The absorbance $(450 \mathrm{~nm})$ was measured in a microplate reader.

2.8. Western Blot Analysis. To obtain whole cell protein extracts, RSC96 cells at day 7 were collected in cell lysis buffer. Whole cell extracts were sonicated and centrifuged to obtain the total proteins. Total protein in each sample was quantitated and concentrations were obtained using the BCA assay (Keygene, Nanjing, China). For Western blot analysis, $25 \mu \mathrm{g}$ of proteins was electrophoretically separated by $10 \%$ SDS-polyacrylamide gel electrophoresis and transferred to nitrocellulose membranes. The membranes were blocked with $5 \%$ bovine serum albumin and incubated with $1-5 \mu \mathrm{g}$ of primary antibodies against Krox-20 and p38/phosphorp38 MAPK. Bound proteins were visualized with HRP-conjugated anti-rabbit IgG for Krox-20, p38, and phospho-p38. 


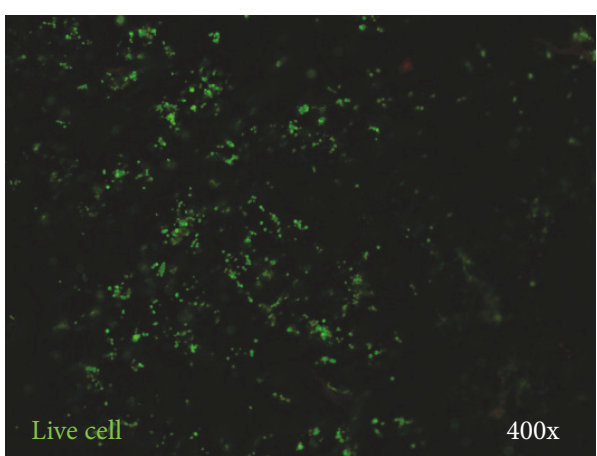

(a) $\mathrm{Col}$

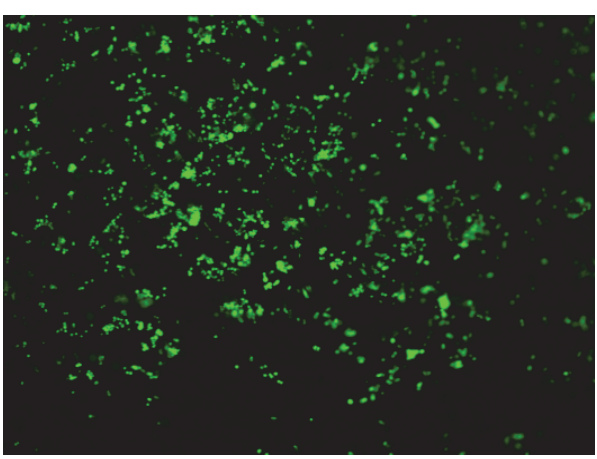

(c) $0.064 \% \mathrm{E}-\mathrm{Col}$

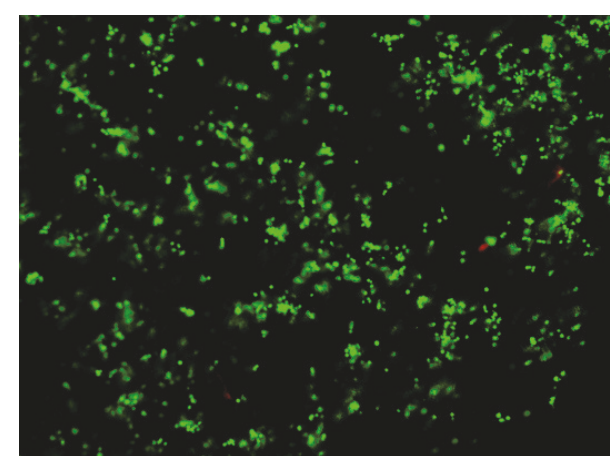

(b) $0.0064 \% \mathrm{E}-\mathrm{Col}$

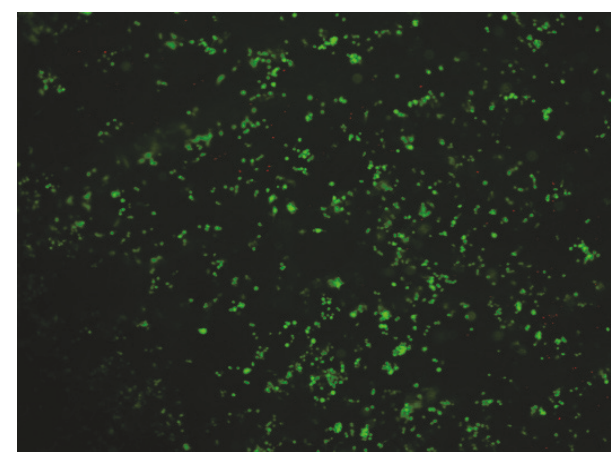

(d) $0.64 \% \mathrm{E}-\mathrm{Col}$

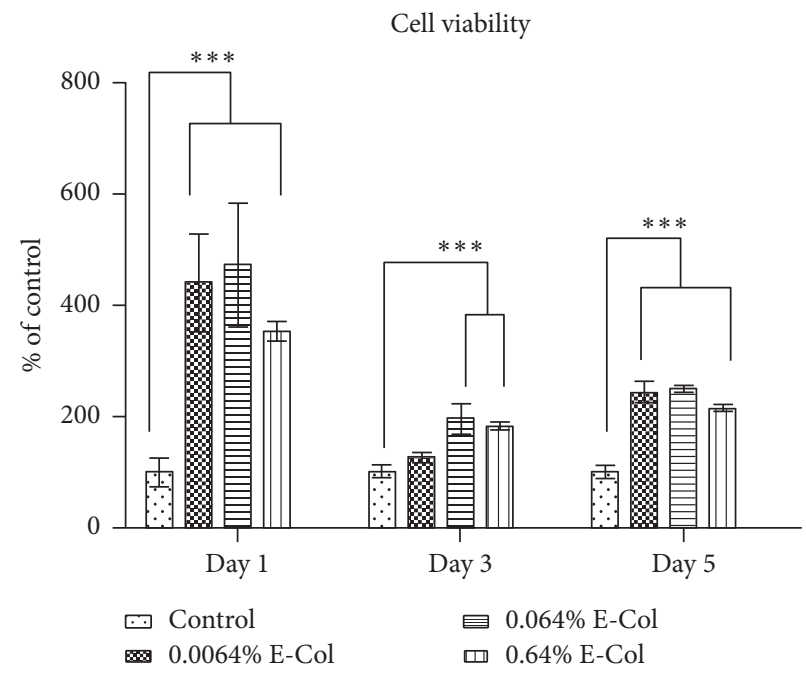

(e)

FIGURE 1: Live staining of RSC96 cells cultured on collagen membranes treated with 0\% EGCG (a), 0.0064\% EGCG (b), 0.064\% EGCG (c), and $0.64 \%$ EGCG (d) on day 5. Live cells appear as green. (e) CCK-8 results of RSC96 cells cultured on various collagen membranes for 1, 3, and 5 days. Culture of different cells of collagen membrane treated or not treated with EGCG revealed the viability of different types of cells. ${ }^{* * *}$ Significant difference compared to control group (Col) at $P<0.001$.

Enhanced chemiluminescence assay was used for the visualization of the protein bands. Moreover, ImageJ was used for the gray level difference analysis of results of Western blot $(N=3)$.

2.9. Statistical Analysis. All results are presented as mean value \pm standard deviation. Differences between groups were analyzed by analysis of variance (one-way ANOVA) followed by Tukey's multiple comparison test $(\alpha=0.05)$ by statistic software GraphPad Prism 5.

\section{Results}

3.1. Cell Viability and Cell Adhesion. Cell viability was assessed with CCK-8 and live cells were stained with CalceinAM. Figure 1(e) shows the results of CCK-8 for RSC96 cells 


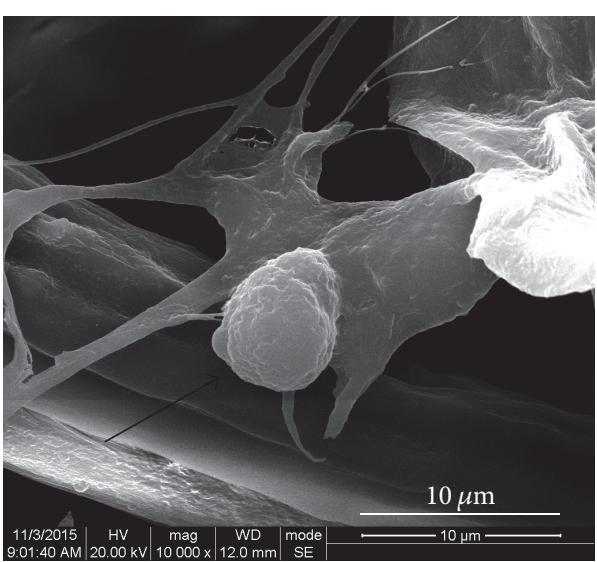

(a) Col

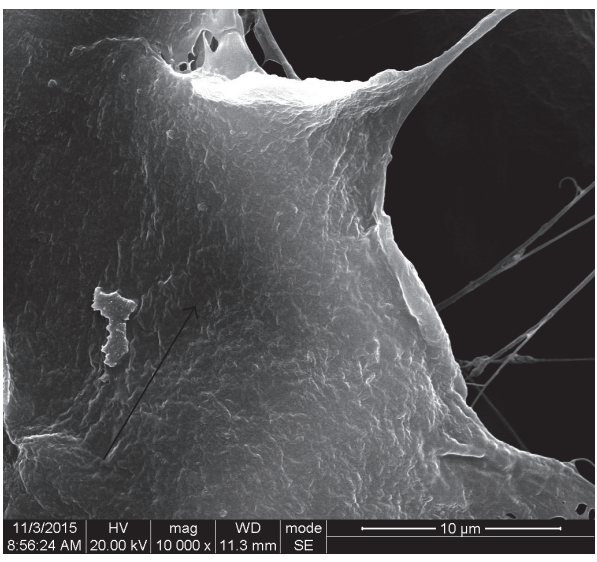

(c) $0.064 \% \mathrm{E}-\mathrm{Col}$

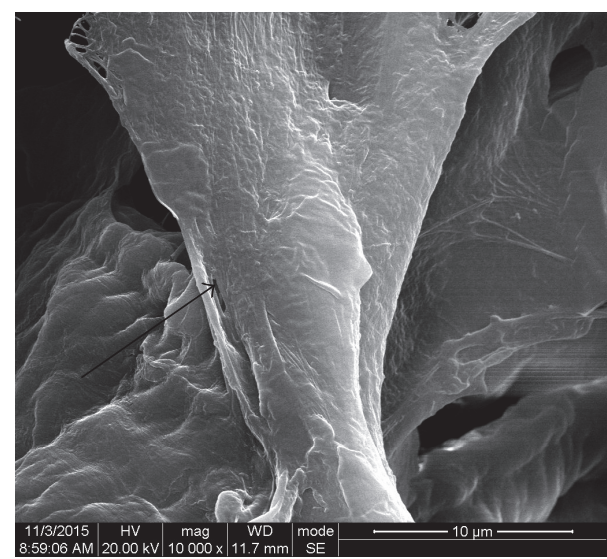

(b) $0.0064 \% \mathrm{E}-\mathrm{Col}$

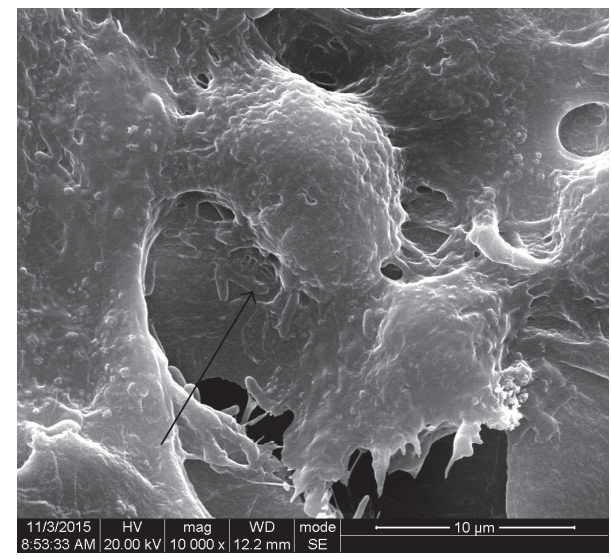

(d) $0.64 \% \mathrm{E}-\mathrm{Col}$

FIGURE 2: SEM images of the morphologies of RSC96 cells adhered to collagen membrane surfaces treated with 0\% EGCG (a), 0.0064\% EGCG (b), 0.064\% EGCG (c), and 0.64\% EGCG (d) after 24 h. 0.064\% EGCG-collagen showed better morphology of RSC96 cells among the experimental groups, indicating higher cell proliferation. The black arrows indicate the cell-surface adhesion.

TABLE 1: Nucleotide primers used for qRT-PCR.

\begin{tabular}{lc}
\hline Genes & Oligonucleotide sequence $\left(5^{\prime}-3^{\prime}\right)$ \\
\hline \multirow{2}{*}{ Gapdh } & Forward: CCGTATCGGACGCCTGGTTA \\
& Reverse: CCGTGGGTAGAGTCATACTGGAAC \\
BDNF & Forward: TCTACGAGACCAAGTGTAATCCCA \\
& Reverse: CTTATGAACCGCCAGCCAAT \\
NGF & Forward: CTGGGCGAGGTGAACATTAACA \\
& Reverse: CAGCCTGTTTGTCGTCTGTTGTC \\
\hline
\end{tabular}

seeded on collagen on days 1,3 , and 5 . Cell viability improved significantly after EGCG addition, whereas 0.064\% EGCGcollagen showed the best effect on promoting proliferation in the experimental groups. In addition, cell viability after addition of $0.064 \%$ EGCG-collagen increased by nearly 5fold compared to that of the control group on day 1 . The images in Figures 1(a)-1(d) were obtained after seeding of RSC96 cells on collagen, with staining carried out on day 5. Live cells were stained green. More live cells were observed on collagen following treatment with the lower concentration of EGCG, whereas the highest concentration of EGCG showed more live cells compared to the control group. SEM analysis (Figure 2) revealed the morphologies of RSC96 cells adhered to different collagen membrane surfaces after $24 \mathrm{~h}$. In EGCGtreated collagen membranes, the RSC96 cells were flatly spread across the sample surfaces. These results indicate that EGCG-collagen promotes the proliferation and cell viability of RSC96 cells.

3.2. Inducing Proliferation and Differentiation of SCs. The levels of neurotrophic factors were associated with improvements in survival, regeneration, differentiation, and synaptogenesis of neural fibers. Figures 3(a)-3(d) show that EGCGtreated collagen significantly increased the expression of neurotrophic factors (BDNF and NGF) secreted by RSC96 cells cultured on collagen, which increased with increasing concentration of EGCG according to RT-PCR and ELISA.

$\mathrm{SC}$ differentiation into a myelinating phenotype requires several transcription factors [23], including Krox-20 proteins which directly regulate myelin protein expression [24, 25]. Figures 4(a)-4(d) show the immunostaining results of Krox20 proteins, which stained as red, while the nucleus stained as 


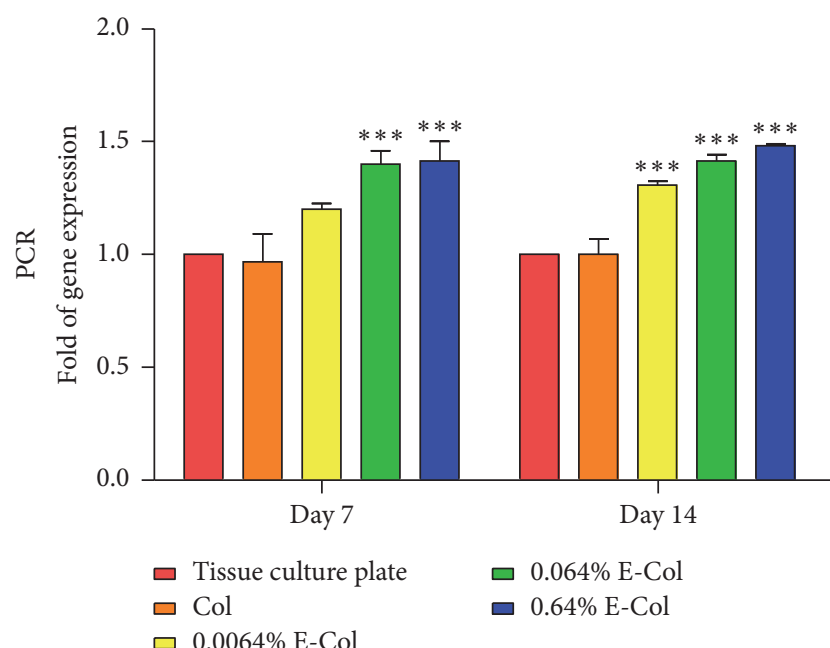

(a)

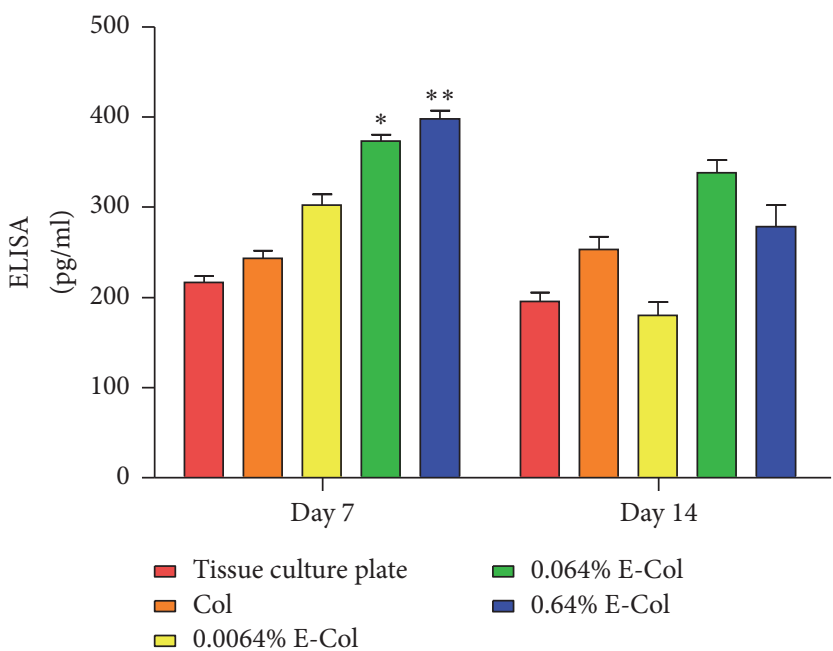

(c)

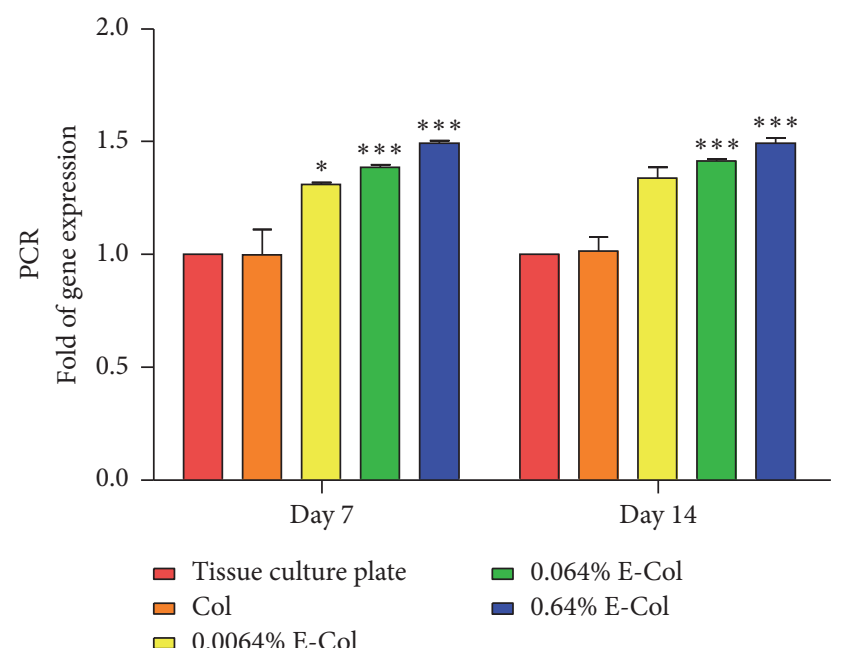

(b)

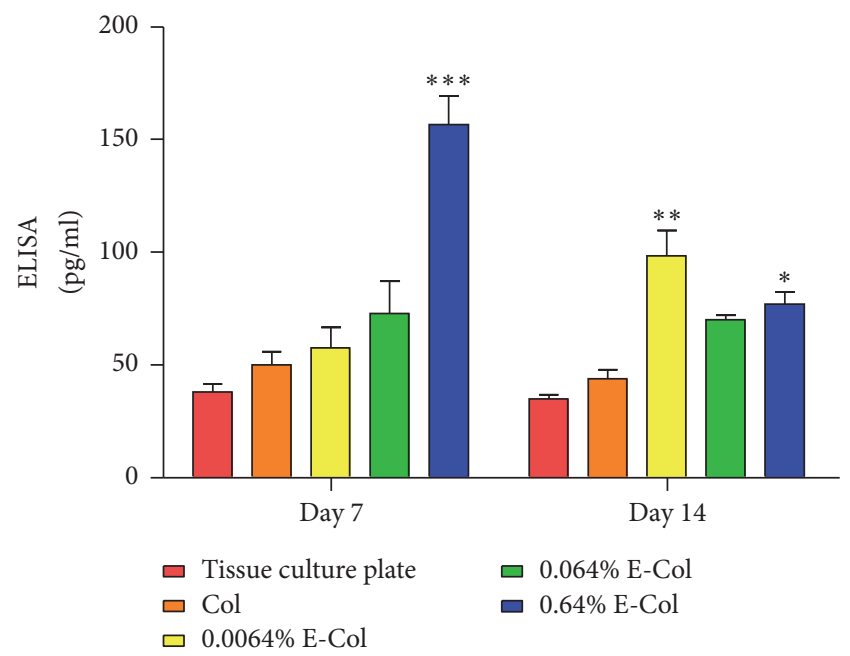

(d)

FIGURE 3: Gene expression of BDGF (a) and NGF (b) in RSC96 cells incubated on EGCG-treated collagen membranes for 7 and 14 days measured by RT-PCR. Quantification of released BDGF (c) and NGF (d) for 7 and 14 days produced by RSC96 cells measured by ELISA. ${ }^{*, * * * * * *}$ Significant difference compared to control group (Col) at $P<0.05, P<0.01$, and $P<0.001$. According to ELISA and RT-PCR, neurotrophic factors produced by RSC96 cells were significantly increased after implantation of EGCG-modified collagen membranes.

blue, indicating that the expression of Krox-20 was increased after EGCG addition. Figure 4(e) shows the results of Western blotting for Krox-20, which also supported increased expression, as the gray levels of Krox-20 in $0.64 \% \mathrm{E}$ and $0.064 \%$ $\mathrm{E}$ were significantly higher than that of the control group. EGCG-modified collagen membranes downregulated P38 MAPK signaling pathways. Phospho-p38 was not activated in the experimental groups but activated in the control group according to Western blotting results (Figure 4(f)). Moreover, reactive oxygen species (ROS) levels were significantly decreased following addition of EGCG. In addition, ROS levels decreased with increasing concentrations of EGCG. Thus, EGCG-treated collagen induced SC differentiation and downregulated ROS levels by downregulating the MAPK P38 signaling pathway.

\section{Discussion}

Because of its biological compatibility and low immunogenicity, an increasing number of studies have shown that collagen can be used to fabricate biomaterials and is useful for nerve reconstruction $[9,10]$. Previous studies have examined the application of EGCG in nerve repair [15-19]. In this study, EGCG-treated collagen induced the proliferation and differentiation of SCs. According to previous studies [22], collagen treated with EGCG showed better mechanical properties and the ability to regulate inflammatory factors, making it a suitable biomaterial for clinical applications.

The CCK-8 and live/dead assay revealed that RSC96 cells treated with EGCG-collagen showed higher cell viability than cells treated with pure collagen. Previous studies suggested 


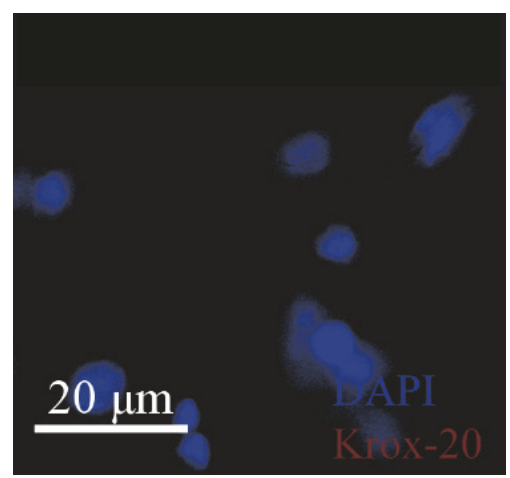

(a) Control

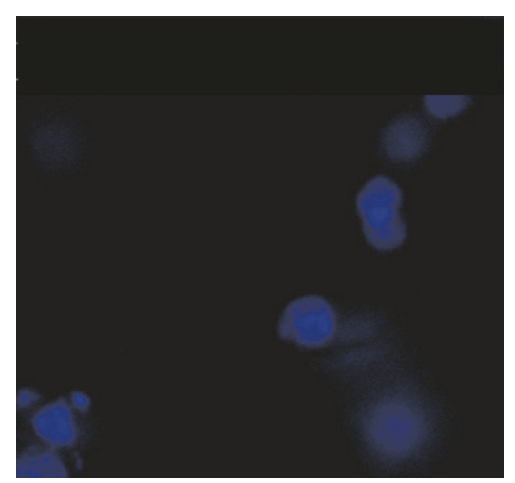

(b) $0.0064 \% \mathrm{E}-\mathrm{Col}$

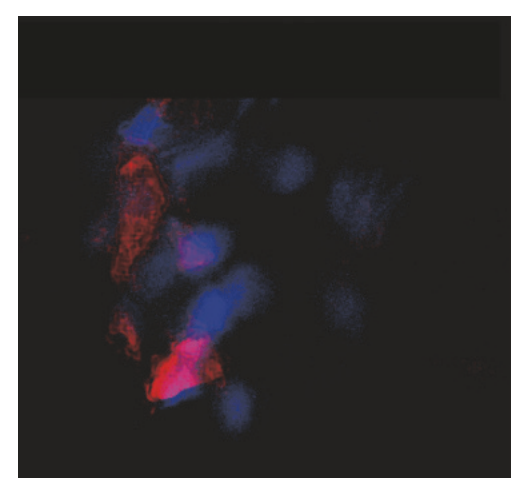

(c) $0.064 \% \mathrm{E}-\mathrm{Col}$

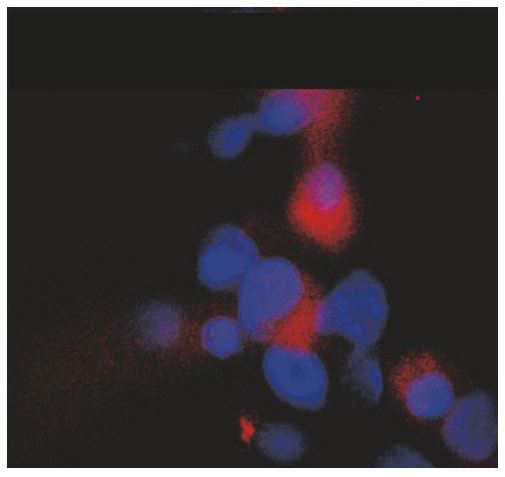

(d) $0.64 \% \mathrm{E}-\mathrm{Col}$

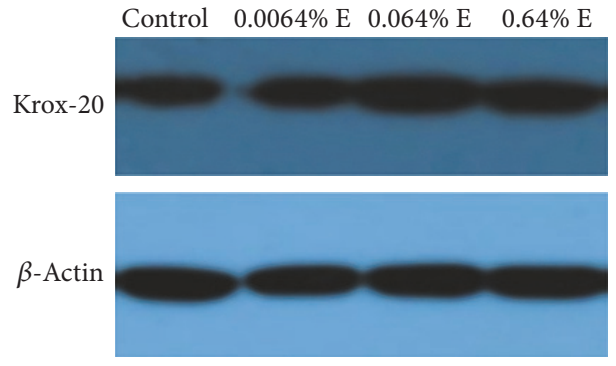

(e)

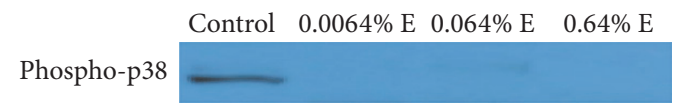

$\beta$-Actin

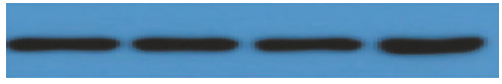

(f)

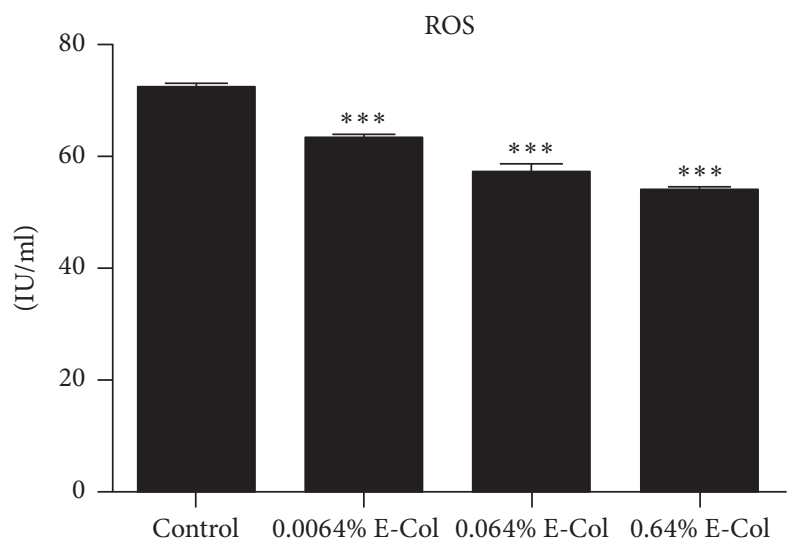

(g)

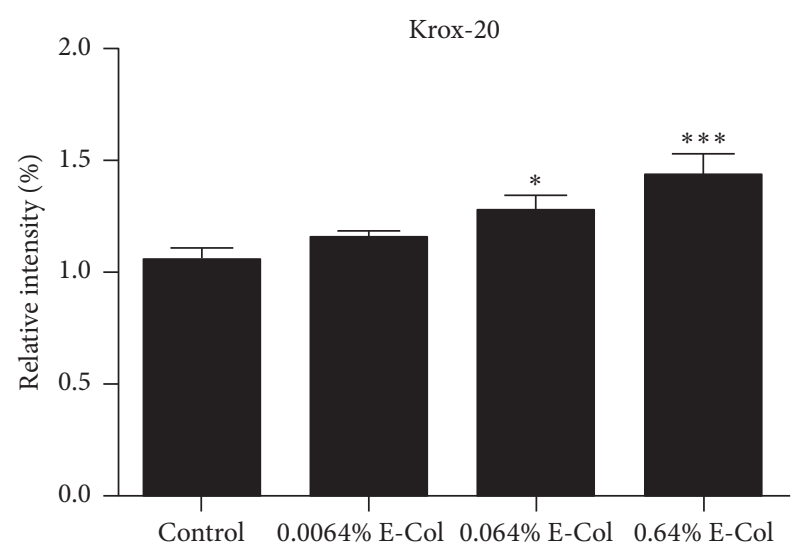

(h)

FIGURE 4: Immunofluorescence staining of Krox-20 (red) and DAPI (blue) in RSC96 cells on collagen membranes treated with 0\% (a), $0.0064 \%$ (b), $0.064 \%$ (c), and $0.64 \%$ (d) EGCG. Compared to the collagen membranes in the control group (a), the collagen membranes treated with different concentrations of EGCG showed increased expression of Krox-20 (f). (e-g) Results of Western blot assay, indicating that EGCG-treated collagen induced Krox-20 expression and decreased ROS levels in RSC96 cells. (h) After gray level difference analysis of Krox-20, the gray levels of $0.64 \% \mathrm{E}$ and $0.064 \% \mathrm{E}$ were significantly higher than that of the control group. ${ }^{*} P<0.05 ;{ }^{* * *} P<0.001$, one-way ANOVA with Tukey's multiple comparison test. 
that EGCG protects nerve cells from oxidative-radical-stressinduced apoptosis [15]. Interestingly, in the present study, the viability of RSC 96 cells significantly increased with increasing concentrations of EGCG, including $0.64 \%$ EGCG, compared to the control group. However, in a previous study, the cell viability of osteoblasts was decreased when the cells were cultured on collagen membranes modified with 0.64\% EGCG [26]. Based on these results, EGCG may have different effects on various cell types. Therefore, the appropriate concentration of EGCG for promoting cell viability varies in different cell types of cells; further studies are needed to confirm this result.

To successfully regenerate nerves, a disconnected nerve must bridge the gap between the proximal and distal stumps, following the SC tube to restore the function of sensory and/or motor targets [3]. Neurotrophic factors support this critical process and improve the survival, regeneration, differentiation, and synaptogenesis of neural fibers after injury. However, it remained unknown whether EGCG crosslinked collagen triggers SC proliferation and differentiation. Therefore, PCR and ELISA were performed to investigate the secretion of neurotrophic factors. The results showed that the expression of neurotrophic factors secreted by RSC96 cells cultured on EGCG-treated collagen were higher than that for untreated collagen, indicating that SCs proliferated and differentiated.

ROS play a deleterious role in the wound healing process, which can be associated with tissue injury. Excessive formation of free radicals can induce cell senescence and apoptosis [27]. The mitogen-activated protein kinase (MAPK) signaling pathway, which is involved in the production of proinflammatory growth factors, plays an important role in signal transduction from the surface to the nucleus of cell [28]. Excess inflammatory factors in the surgical site may lead to complications around implant biomaterials, causing regeneration failure $[29,30]$. According to our experiment results, the EGCG-modified collagen membrane downregulated the levels of ROS. Moreover, the membrane inhibited activation of the MAPK P38 signaling pathway caused by ROS.

The differentiation of SCs into a myelinating phenotype requires several transcription factors, including Krox-20, a zinc-finger transcription factor essential for peripheral myelination [31]. Krox-20 also directly regulates myelin protein expression $[24,25]$. In the present study, expression of Krox-20, determined by immunostaining, increased after the addition of EGCG in a dose-dependent manner, further indicating the differentiation of SCs into a myelinating phenotype. The increased expression of Krox-20 was also detected by Western blotting; the gray levels of $0.64 \% \mathrm{E}$ and $0.064 \%$ E were significantly higher than that of the control group. These results indicate that EGCG-modified collagen membranes are innovative immune-mediated biomaterials that can be used to guide tissue regeneration.

\section{Conclusions}

In this study, collagen cross-linked by EGCG showed smooth surfaces and more uniform fibers. In addition, collagen membranes treated with appropriate concentration of EGCG can improve SC proliferation, differentiation, and migration and downregulate ROS levels by downregulating the MAPK p38 signaling pathway, indicating their potential for use in GTR treatment. This material may be used in nerve repair and numerous other clinical applications.

\section{Additional Points}

Highlights. EGCG-treated collagen membrane significantly induces RSC96 cell proliferation and differentiation in vitro. EGCG-treated collagen membrane significantly induces secretion of neurotrophic factors in RSC96 cells. EGCG-treated collagen membrane downregulates ROS level in RSC96 cells by downregulating MAPK P38 signaling pathway.

\section{Conflicts of Interest}

The authors declare no potential conflicts of interest with respect to the authorship and/or publication of this article.

\section{Acknowledgments}

This work was supported by grants from the National Natural Science Foundation of China (no. 81671023) and the National Key Research Development Program of China (nos. 2016YFA0201703/2016YFA0201700).

\section{References}

[1] K. A. Black, B. F. Lin, E. A. Wonder et al., "Biocompatibility and characterization of a peptide amphiphile hydrogel for applications in peripheral nerve regeneration," Tissue Engineering Part A, vol. 21, no. 7-8, pp. 1333-1342, 2015.

[2] W. Z. Ray and S. E. Mackinnon, "Management of nerve gaps: autografts, allografts, nerve transfers, and end-to-side neurorrhaphy," Experimental Neurology, vol. 223, no. 1, pp. 7785, 2010.

[3] L. Jiang, S. Jones, and X. Jia, "Stem cell transplantation for peripheral nerve regeneration: Current options and opportunities," International Journal of Molecular Sciences, vol. 18, no. 1, article no. 94, 2017.

[4] J. N. Glenn, M. E. Miner, and L. F. Peltier, "The treatment of fractures of the femur in patients with head injuries," Clinical Orthopaedics and Related Research, vol. 2004, no. 422, pp. 142144, 1973.

[5] T. Yokoyama, T. Saino, N. Nakamuta, and Y. Yamamoto, "Topographic distribution of serotonin-immunoreactive urethral endocrine cells and their relationship with calcitonin gene-related peptide-immunoreactive nerves in male rats," Acta Histochemica, vol. 119, no. 1, pp. 78-83, 2017.

[6] P. Tonelli et al., "Bone regeneration in dentistry," Clinical Cases in Mineral and Bone Metabolism, vol. 8, no. 3, pp. 24-28, 2011.

[7] H. Wang and L. Boyapati, “"pASS” principles for predictable bone regeneration," Implant Dentistry, vol. 15, no. 1, pp. 8-17, 2006.

[8] C. Chu et al., "Nanoparticles combined with growth factors: recent progress and applications," RSC Advances, vol. 6, no. 93, pp. 90856-90872, 2016.

[9] D. J. Macaya, K. Hayakawa, K. Arai, and M. Spector, "Astrocyte infiltration into injectable collagen-based hydrogels containing 
FGF-2 to treat spinal cord injury," Biomaterials, vol. 34 , no. 14 , pp. 3591-3602, 2013.

[10] Y. Cui, C. Lu, D. Meng et al., "Collagen scaffolds modified with CNTF and bFGF promote facial nerve regeneration in minipigs," Biomaterials, vol. 35, no. 27, pp. 7819-7827, 2014.

[11] K. Sadtler, K. Estrellas, B. W. Allen et al., "Developing a proregenerative biomaterial scaffold microenvironment requires $\mathrm{T}$ helper 2 cells," Science, vol. 352, no. 6283, pp. 366-370, 2016.

[12] Y. Zhong, Y.-S. Chiou, M.-H. Pan, and F. Shahidi, "Antiinflammatory activity of lipophilic epigallocatechin gallate (EGCG) derivatives in LPS-stimulated murine macrophages," Food Chemistry, vol. 134, no. 2, pp. 742-748, 2012.

[13] S. Riegsecker, D. Wiczynski, M. J. Kaplan, and S. Ahmed, "Potential benefits of green tea polyphenol EGCG in the prevention and treatment of vascular inflammation in rheumatoid arthritis," Life Sciences, vol. 93, no. 8, pp. 307-312, 2013.

[14] M. Nakamuta et al., "Epigallocatechin-3-gallate, a polyphenol component of green tea, suppresses both collagen production and collagenase activity in hepatic stellate cells," International Journal of Molecular Medicine, vol. 16, no. 4, pp. 677-681, 2005.

[15] S.-H. Koh, S. H. Kim, H. Kwon et al., "Epigallocatechin gallate protects nerve growth factor differentiated PC12 cells from oxidative-radical-stress-induced apoptosis through its effect on phosphoinositide 3-kinase/Akt and glycogen synthase kinase3," Molecular Brain Research, vol. 118, no. 1-2, pp. 72-81, 2003.

[16] J. Xie, L. Jiang, T. Zhang, Y. Jin, D. Yang, and F. Chen, "Neuroprotective effects of Epigallocatechin-3-gallate (EGCG) in optic nerve crush model in rats," Neuroscience Letters, vol. 479, no. 1, pp. 26-30, 2010.

[17] W. M. Renno, M. Al-Maghrebi, A. Alshammari, and P. George, "(-)-Epigallocatechin-3-gallate (EGCG) attenuates peripheral nerve degeneration in rat sciatic nerve crush injury," Neurochemistry International, vol. 62, no. 3, pp. 221-231, 2013.

[18] X. Xifró, L. Vidal-Sancho, P. Boadas-Vaello et al., "Novel epigallocatechin-3-gallate (EGCG) derivative as a new therapeutic strategy for reducing neuropathic pain after chronic constriction nerve injury in mice," PLoS ONE, vol. 10, no. 4, Article ID e0123122, 2015.

[19] W. Renno, K. Khan, and L. Benov, "Beneficial effect of ()-epigallocatechin-3-gallate (EGCG) on sciatic nerve crush injury: stereological analysis," The FASEB Journal, vol. 29, supplement 1, 2015.

[20] C. Chu et al., "Collagen membrane and immune response in guided bone regeneration: recent progress and perspectives," Tissue Engineering Part B: Reviews, 2017.

[21] C. Chu et al., "Evaluation of nano hydroxyapaptite (nanoHA) coated epigallocatechin-3-gallate (EGCG) cross-linked collagen membranes," Materials Science and Engineering: $C$, vol. 78, pp. 258-264, 2017.

[22] C. Chu, J. Deng, L. Xiang et al., "Evaluation of epigallocatechin3-gallate (EGCG) cross-linked collagen membranes and concerns on osteoblasts," Materials Science and Engineering C, vol. 67, pp. 386-394, 2016.

[23] J. Svaren and D. Meijer, "The molecular machinery of myelin gene transcription in Schwann cells," Glia, vol. 56, no. 14, pp. 1541-1551, 2008.

[24] S. E. LeBlanc, S.-W. Jang, R. M. Ward, L. Wrabetz, and J. Svaren, "Direct regulation of myelin protein zero expression by the Egr2 transactivator," Journal of Biological Chemistry, vol. 281, no. 9, pp. 5453-5460, 2006.
[25] R. Nagarajan, J. Svaren, N. Le, T. Araki, M. Watson, and J. Milbrandt, "EGR2 mutations in inherited neuropathies dominantnegatively inhibit myelin gene expression," Neuron, vol. 30, no. 2, pp. 355-368, 2001.

[26] C. Chenyu, J. Deng, Y. Hou et al., "Application of PEG and EGCG modified collagen-base membrane to promote osteoblasts proliferation," Materials Science and Engineering C, vol. 76, pp. 31-36, 2017.

[27] M. Valko, D. Leibfritz, J. Moncol, M. T. D. Cronin, M. Mazur, and J. Telser, "Free radicals and antioxidants in normal physiological functions and human disease," International Journal of Biochemistry and Cell Biology, vol. 39, no. 1, pp. 44-84, 2007.

[28] J. M. Kyriakis and J. Avruch, "Mammalian MAPK signal transduction pathways activated by stress and inflammation: a 10 -year update," Physiological Reviews, vol. 92, no. 2, pp. 689737, 2012.

[29] N. Broggini, L. M. McManus, J. S. Hermann et al., "Peri-implant inflammation defined by the implant-abutment interface," Journal of Dental Research, vol. 85, no. 5, pp. 473-478, 2006.

[30] T. Albrektsson et al., "Is marginal bone loss around oral implants the result of a provoked foreign body reaction?" Clinical Implant Dentistry \& Related Research, vol. 16, article 155, no. 2, 2014.

[31] K. Venken, E. Di Maria, E. Bellone et al., "Search for mutations in the EGR2 corepressor proteins, NAB1 and NAB2, in human peripheral neuropathies," Neurogenetics, vol. 4, no. 1, pp. 37-41, 2002 . 

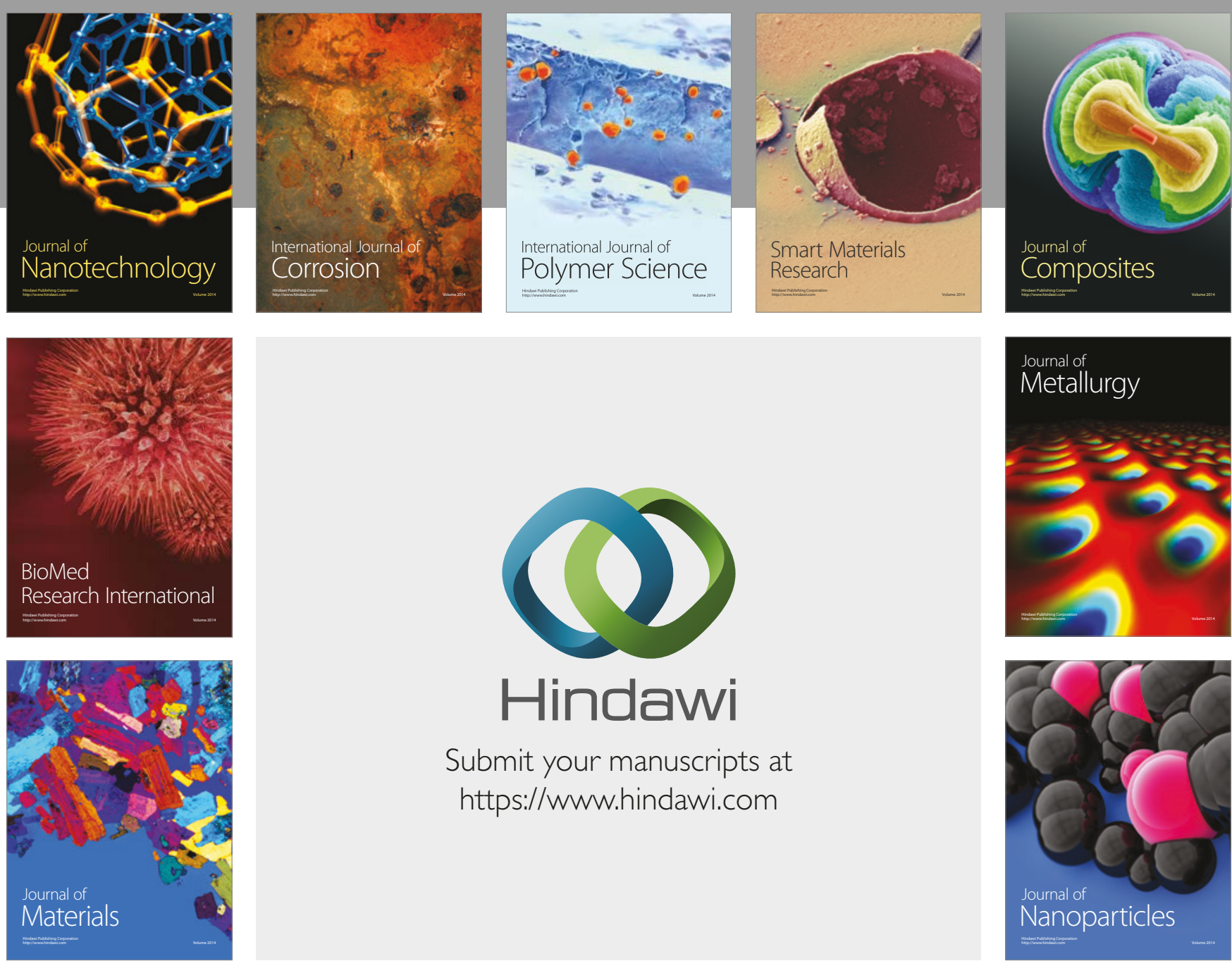

\section{Hindawi}

Submit your manuscripts at

https://www.hindawi.com
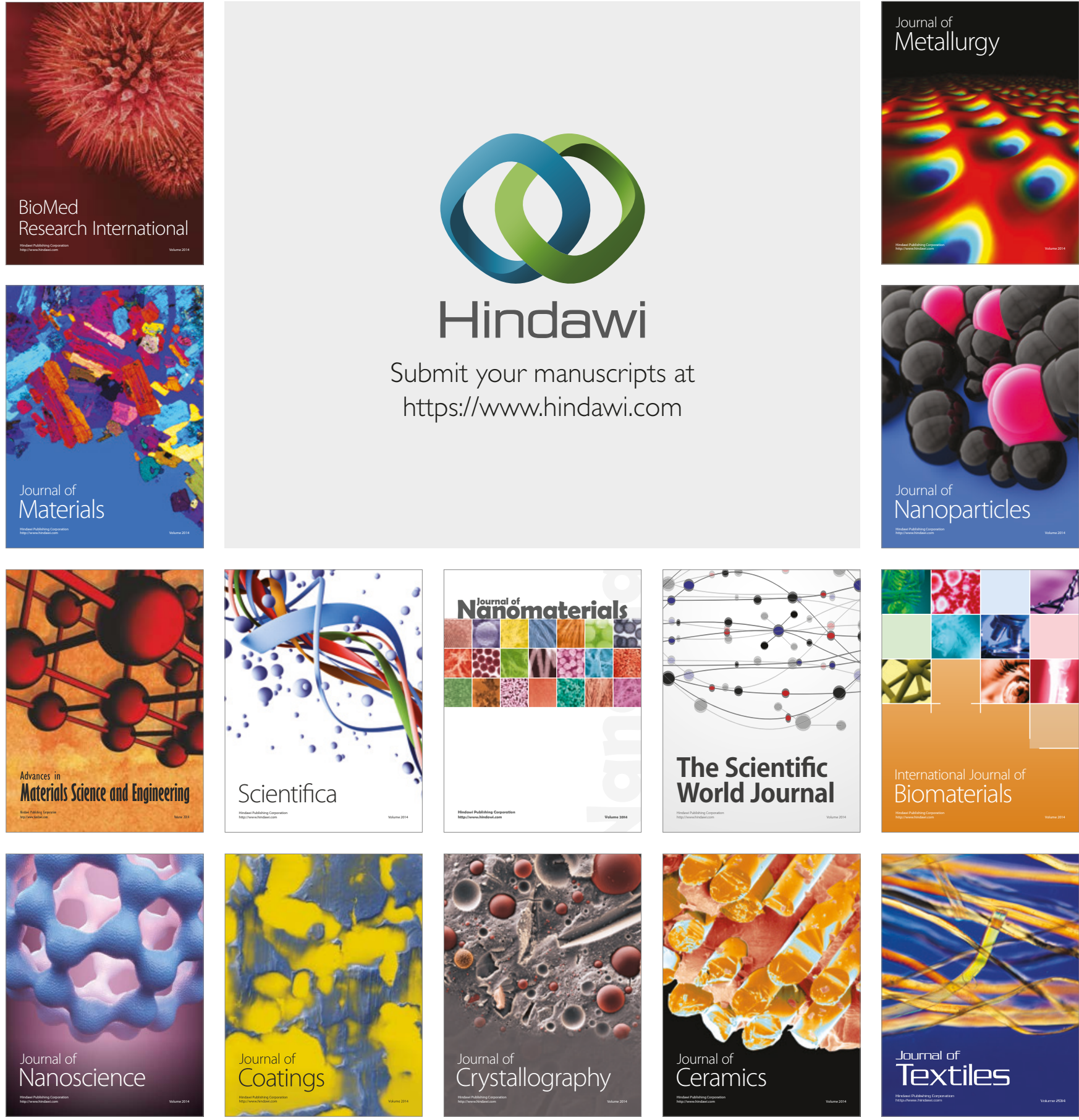

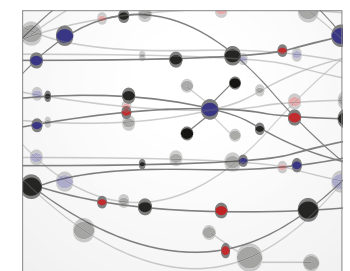

The Scientific World Journal
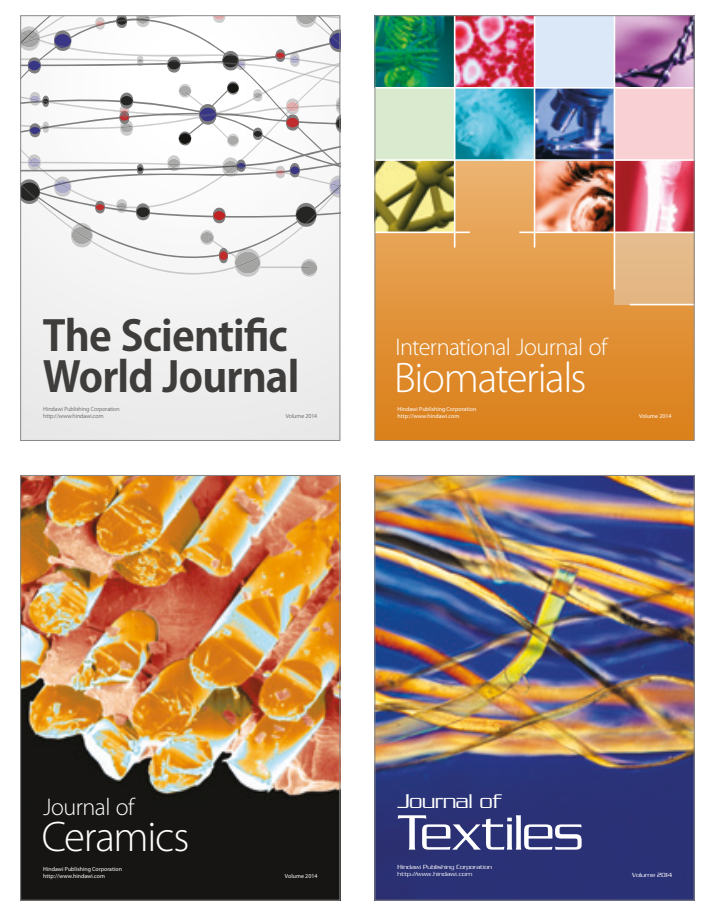